\title{
VALERIANA CORYNODES (CAPRIFOLIACEAE), NUEVA ESPECIE PARA LA FLORA DE CHILE
}

\author{
Sebastián Teillier ${ }^{1}$, Rosemarie Lund ${ }^{2}$ \& Fernando O. Zuloaga ${ }^{3}$ \\ ${ }^{1}$ Universidad Central de Chile, Escuela de Arquitectura y Paisaje, Santa Isabel 1117, Santiago de Chile, Chile; \\ steillier@gmail.com (autor corresponsal). \\ ${ }^{2}$ SGA Consultores ambientales, Rosario Norte 100 Piso 7, Las Condes, Santiago de Chile, Chile. \\ ${ }^{3}$ Instituto de Botánica Darwinion (CONICET-ANCEFN), Labardén 200, Casilla de Correo 22, B1642HYD, San Isidro, \\ Buenos Aires, Argentina.
}

Abstract. Teillier, S.; R. Lund \& F. O. Zuloaga. 2020. Valeriana corynodes (Caprifoliaceae), a new species for the Flora of Chile. Darwiniana, nueva serie 8(1): 410-413.

We report here a new species of Valeriana for the Chilean flora. Valeriana corynodes, a species up to now considered endemic of Argentina, province of San Juan. The species is here cited for the region of Atacama, in quebrada La Brea in the upper river Copiapó. We provided here a description, an illustration, a geographic distribution map, and a key of related Valeriana species in Chile.

Keywords. Caprifoliaceae; Chile; taxonomy; Valeriana.

Resumen. Teillier, S.; R. Lund \& F. O. Zuloaga. 2020. Valeriana corynodes (Caprifoliaceae), nueva especie para la Flora de Chile. Darwiniana, nueva serie 8(1): 410-413.

En este trabajo se reporta una nueva especie de Valeriana para la flora de Chile. Se trata de Valeriana corynodes, una especie considerada hasta ahora como endémica de la provincia de San Juan, Argentina. Ejemplares de esta especie se registraron en la quebrada La Brea, en la cordillera de la Región de Atacama, en la cuenca alta del río Copiapó. Se describe e ilustra la especie, se presenta un mapa con la distribución conocida y se provee una clave para diferenciar a las especies afines de Valeriana en Chile.

Palabras clave. Caprifoliaceae; Chile; taxonomía; Valeriana.

\section{INTRODUCCIÓN}

El género Valeriana L., originalmente incluido en la familia Valerianaceae, se clasifica en la actualidad en la familia Caprifoliaceae, subfamilia Valerianoideae Raf. (APG IV, 2016), la que integra junto con el género Valerianella Mill. Valeriana reúne unas 270 (APG IV, 2016) a 250 especies en todo el mundo (Kutschker, 2019), las que en su mayoría crecen en el hemisferio norte, con un importante centro secundario de diversificación en la región andina de Sudamérica.

En Chile, las especies de Valeriana, quizá debido a su gran número y complejidad, han sido revisadas en escasas ocasiones. $\mathrm{Al}$ respecto, destacan el aporte de K. Reiche (1902), con su revisión de la flora de
Chile, una contribución posterior con la revisión de todas las especies de Chile por parte de la botánica argentina O. Borsini (1966) y finalmente, una revisión de las especies del centro y sur llevada a cabo por A. Kutschker (2011). Producto de estas revisiones y hallazgos como el de Valeriana moyanoi Speg. en la Patagonia de Aisén (Saldivia \& Rojas, 2006), se reportan en Chile 42 especies del género (Rodríguez et al., 2018), siendo 17 endémicas de Chile y 25 nativas.

En una excursión realizada por uno de los autores en junio de 2018 a la cordillera de Copiapó, Región de Atacama, se registró a Valeriana corynodes Borsini, lo que resultó un hallazgo nuevo para la flora vascular de Chile. En este trabajo se describe la especie teniendo como referencia a Kutschker (2019). 


\section{TRATAMIENTO TAXONÓMICO}

Valeriana corynodes Borsini, Gen. Sp. Pl. Argent. 2: 341, tab. 124b, 148a. 1944. TIPO: Argentina. San Juan. Cordillera de Colangüil, quebrada El Salto, 16 enero 1930. R. A. Pérez Moreau s.n. (holotipo, BA!).

Hierba perenne, forma cojines densos, cespitosos. Rizoma hasta de $5 \mathrm{~cm}$ de longitud, leñoso, ramificado. Tallo aéreo muy breve, densamente pubescente, con los restos de las hojas secas en la base, los floríferos hasta de $15 \mathrm{~cm}$ de alto. Hojas basales pecioladas, dispuestas en rosetas, láminas pinnatisectas hasta de $8 \mathrm{~cm}$ de longitud, imparipinnadas, con 5-7 pares de folíolos laterales sésiles, hasta de $1 \mathrm{~cm}$ de longitud, irregulares, ovados, lanceolados, obtusos o agudos; folíolo terminal \pm simétrico. Hojas caulinares pequeñas. Inflorescencia terminal, espiciforme; brácteas de 3-5 mm de largo; bractéolas lanceoladas o linear-lanceoladas, de 2-3 mm de longitud. Flores perfectas, de 3-5 $\mathrm{mm}$ de longitud; cáliz corto, corola infundibuliforme, de 2-2,5 $\mathrm{mm}$ de longitud, blanca, pubescente; estambres inclusos, de 1-1,4 mm de longitud; anteras oblongas; ovario de 1-1,4 mm de longitud, estilo filiforme, exerto. Frutos angostamente ovoides o claviformes, de 4 $\mathrm{mm}$ de longitud, pubescentes, papilosos en la base; cáliz no desarrollado en un papus plumoso. Fig. 1.

Distribución y ecología. La especie fue descrita a partir de material coleccionado por R. A. Pérez Moreau en la cordillera del Colangüil, provincia de San Juan y hasta ahora se consideró como endémica de Argentina y de dicha provincia. En la provincia de San Juan, la especie crece en la vegetación puneña entre los 2200 y 3900 m s.m. (Zuloaga et al., 2008; 2019; Weberling, 2017; Kutschker, 2019). Además, Weberling (2017) cita a la especie para la provincia de Mendoza, en la localidad de Las Heras, sin embargo, no constan colecciones en esa provincia.

Los ejemplares chilenos de la especie se encontraron en una quebrada, a unos $3330 \mathrm{~m}$ de altitud (Fig. 2). Al parecer, al menos en el
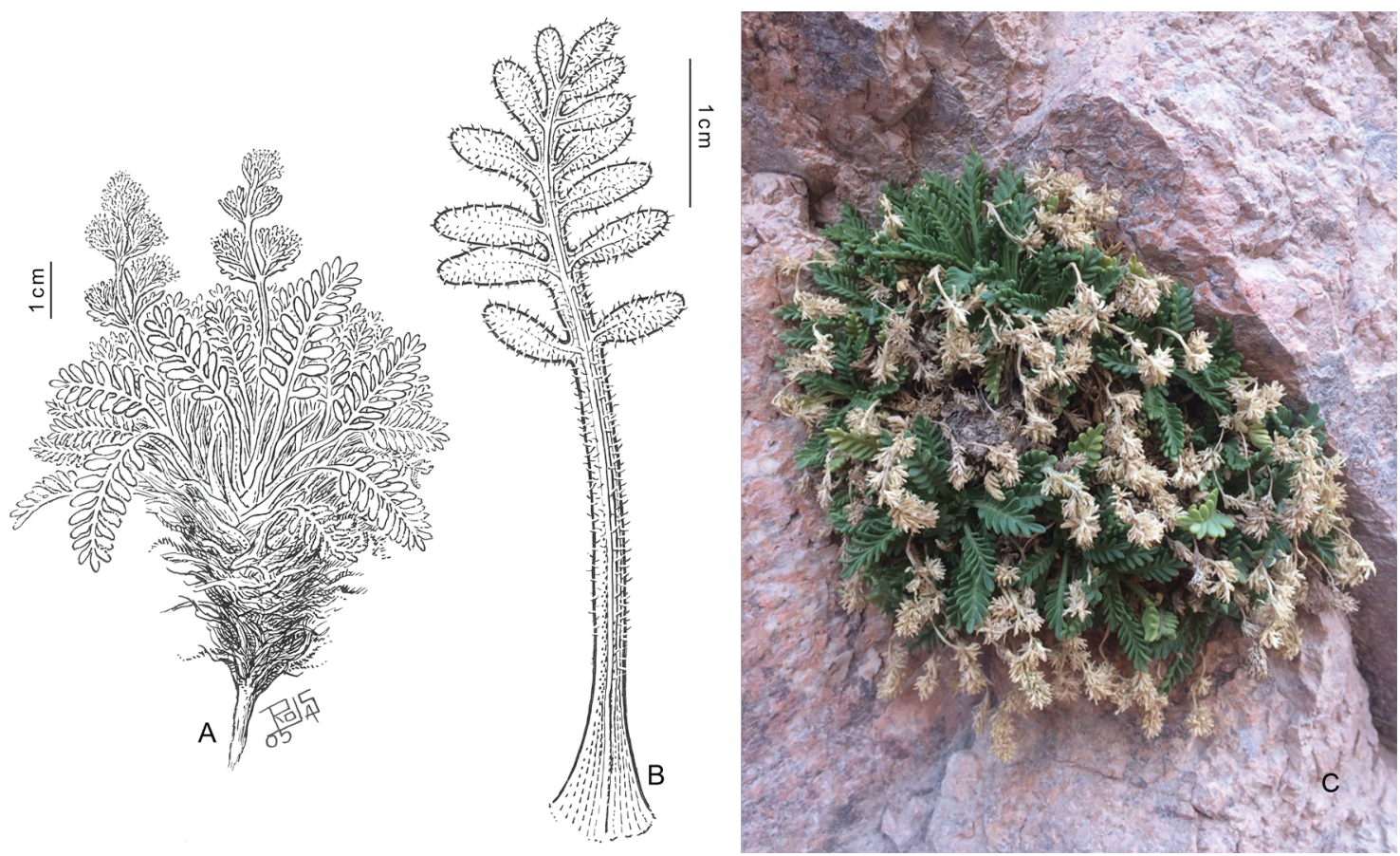

Fig. 1. Valeriana corynodes Borsini. A, planta en flor. B, hoja. Fuente: Kutschker (2019). C, ejemplar de Valeriana corynodes, fotografiado en la quebrada La Brea, cordillera de Copiapó, Región de Atacama, Chile. Fotografía R. Lund. Figura en color en la versión en línea http://www.ojs.darwin.edu.ar/index.php/darwiniana/article/view/897/1187 
área del hallazgo, sería una especie que crece sobre las paredes rocosas que mantienen mejor la humedad, característica coincidente con algunas notas escritas en ejemplares de Argentina, como por ejemplo en Pujalte 16 (SI). En el área es una especie escasa y crece en sitios de difícil acceso, lo que explica que haya pasado inadvertida hasta ahora.

En los Andes de Atacama crecen, además de $V$. corynodes, $V$. nivalis Wedd. y V. stricta Clos (Fig. 3A y 3B). A continuación se provee una clave para determinarlas:

1. Hierbas con tallo aéreo, hojas basales y caulinares bien definidas. Tallos floríferos de 20-100 cm de altura Valeriana stricta 1. Hierbas acaules, hojas dispuestas en roseta basal. Tallos floríferos de hasta $15 \mathrm{~cm}$ de altura 2 2 (1). Hojas enteras, glabras; inflorescencia globosa, frutos con papus Valeriana nivalis 2. Hojas pinnatipartidas, cortamente pubescentes; inflorescencia espiciforme; frutos sin papus

Valeriana corynodes

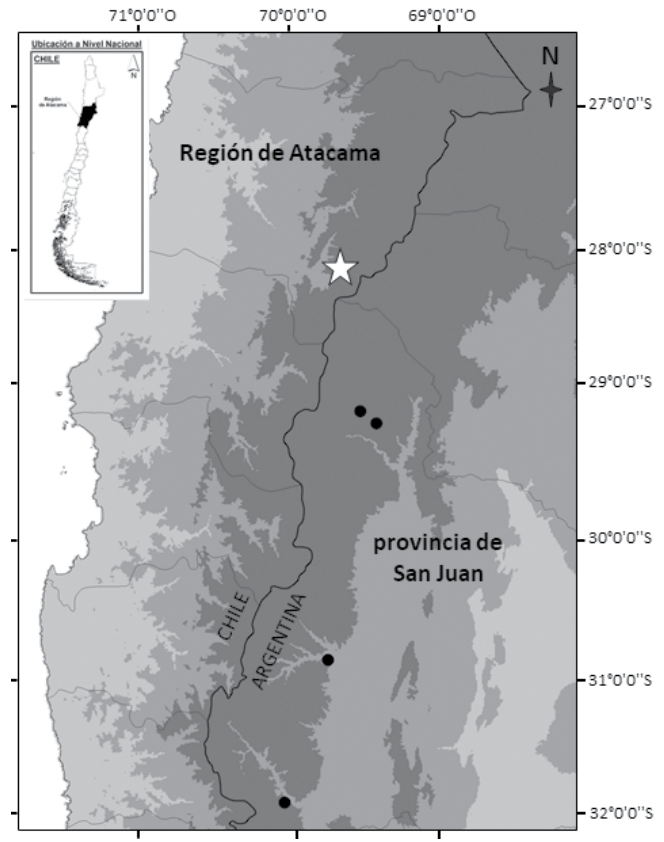

Fig. 2. Ubicación del ejemplar de Valeriana corynodes en la Región de Atacama (estrella); los puntos negros corresponden a las localidades conocidas de la especie en Argentina.
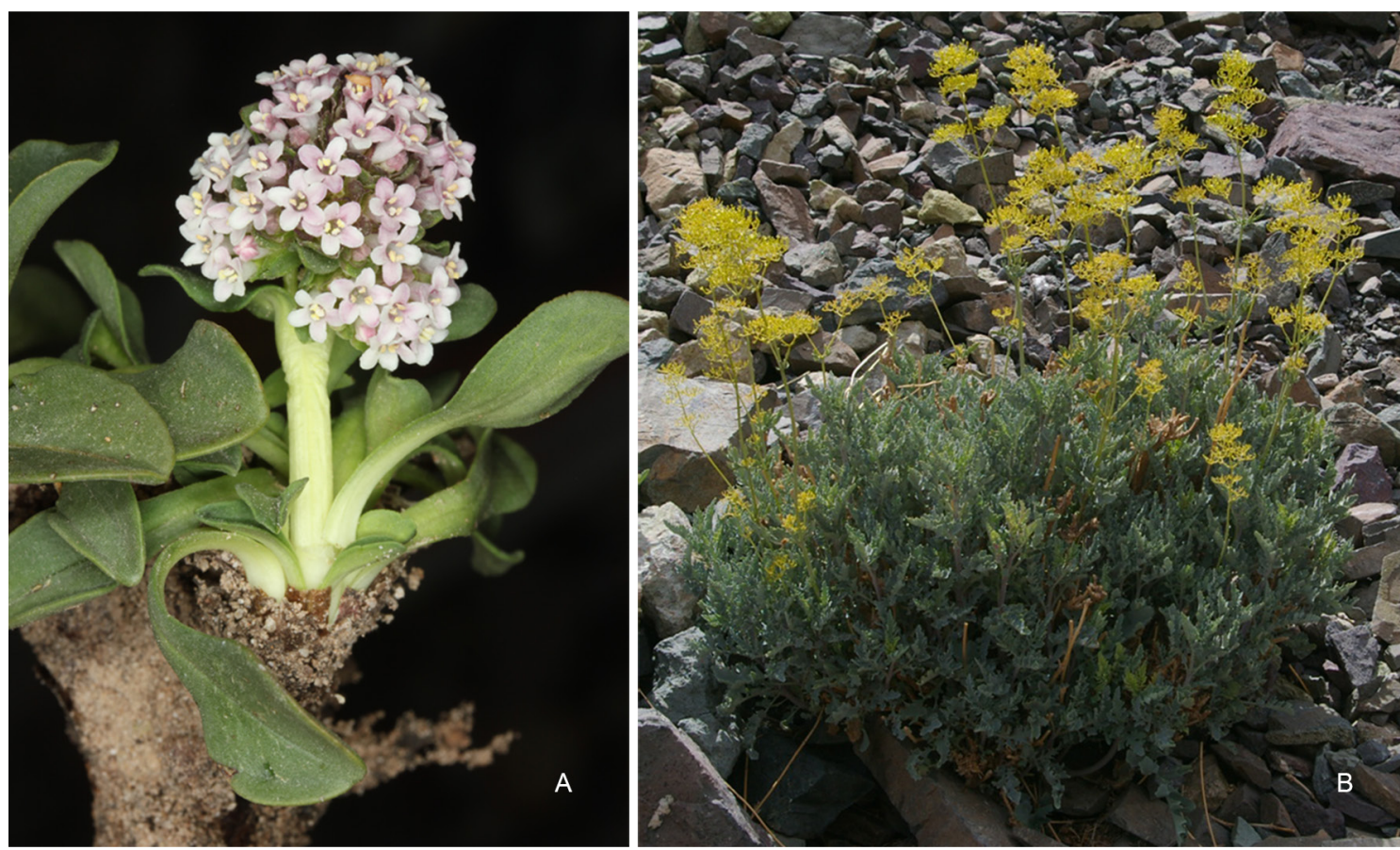

Fig. 3. A, ejemplar de $V$. nivalis (Zuloaga et al. 14361, Argentina. Fotografía C. A. Zanotti). B, ejemplar de $V$. stricta (Teillier \& Riveros 8229, Chile. Fotografia S. Teillier). Figura en color en la versión en línea http://www.ojs.darwin.edu.ar/ index.php/darwiniana/article/view/897/1187 


\section{Material examinado}

CHILE. Región de Atacama: provincia de Copiapó, comuna de Tierra Amarilla, quebrada La Brea, 2809'01' S, 69³6'18' O, 3330 m s.m., 27VI-2018, R. Lund \& F. Osorio 001 (CONC).

ARGENTINA. San Juan. Dpto. Calingasta: Portezuelo de la Ollita, borde de vega, 25-I-1991, Kiesling 7712 (SI); camino de Barreal a El Pachón, al E de Laguna Blanca, 26-I-2018, Salariato et al. 295 (SI). Dpto. Iglesia: Reserva de San Guillermo, cerro Imán, 9-XII-1981, Pujalte 16 (SI); Parque Nacional San Guillermo, puesto Agua del Godo, 23-II-1981, Nicora 8328 (SI).

\section{AGRADECIMIENTOS}

El primer autor agradece a SGA S.A. por las facilidades para acceder al terreno, a F. Osorio y D. Titichoca que ayudaron en los trabajos de terreno y a A. Soto, por la figura con el mapa del hallazgo.

\section{BIBLIOGRAFÍA}

APG IV. Byng, J. W. et al. (compiladores). 2016. An update of the Angiosperm Phylogeny Group classification for the orders and families of flowering plants. Botanical Journal of the Linnean Society 181: 1-20.
Borsini, O. E. 1966. Valerianáceas de Chile. Lilloa 32: 375-476. Kutschker, A. 2011. Revisión del género Valeriana (Valerianaceae) en Sudamérica austral. Gayana Botánica 68(2): 244-296.

Kutschker, A. 2019. Caprifoliaceae. En F. O. Zuloaga \& M. Belgrano (eds.), Flora Argentina 20(2): 211-262.

Reiche, K. 1902. Estudios críticos sobre la flora de Chile. Flora de Chile 3:1-425.

Rodríguez, R.; C. Marticorena, D. Alarcón, C. Baeza, L. Cavieres, V. Finot, N. Fuentes, A. Kiessling, M. Mihoc, A. Pauchard, E. Ruiz, P. Sánchez \& A. Marticorena. 2018. Catálogo de las plantas vasculares de Chile. Gayana Botánica 75(1): 1-430.

Saldivia, P. \& G. Rojas. 2006. Valeriana moyanoi Speg. (Valerianaceae), new record for the Chilean flora. Gayana Botánica 63(2): 183-186.

Weberling, F. 2017. Valerianaceae. En R. Kiesling (ed.), Flora de San Juan 3a: 285-291.

Zuloaga, F. O.; O. Morrone, M. J. Belgrano, C. Marticorena \& E. Marchesi. (eds.) 2008. Catálogo de las plantas vasculares del Cono Sur. Monographs in Systematic Botany from the Missouri Botanical Garden 107: 3 Vols., 3348 p. Base de datos asociada en INTERNET: http://www.darwin.edu. ar/Proyectos/FloraArgentina/FA.asp (Consultada hasta 06/2020).

Zuloaga, F. O.; M. J. Belgrano \& C. A. Zanotti. 2019. Actualización del catálogo de plantas vasculares del Cono Sur. Darwiniana. nueva serie 7(2): 208-278. DOI: https:// doi.org/10.14522/darwiniana.2019.72.861 